S. SINGHA, T. PATRA, C. G. DANILIUC, F. GLORIUS* (WESTFÄLISCHE WILHELMS-

UNIVERSITÄT MÜNSTER, GERMANY)

Highly Enantioselective [5+2] Annulations through Cooperative N-Heterocyclic Carbene (NHC) Organocatalysis and

Palladium Catalysis

J. Am. Chem. Soc. 2018, 140, 3551-3554.

\section{Enantioselective [5+2] Annulations with a Cooperative Catalytic System}

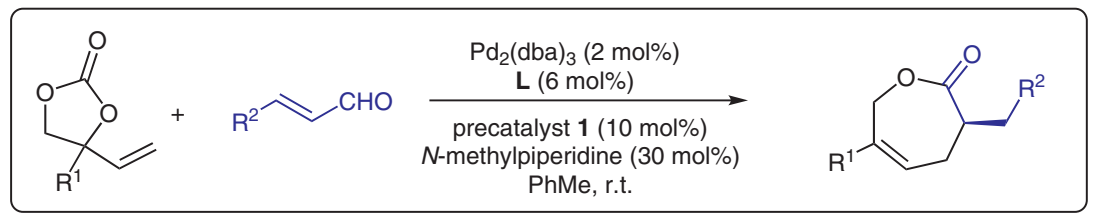

\begin{abstract}
Representative examples:
\end{abstract}<smiles>O=C1OCC(c2ccccc2)=CCC1Cc1ccccc1</smiles><smiles>CC(C)CC1CC=C(Cc2ccccc2)COC1=O</smiles><smiles>O=C1OCC(c2ccco2)=CCC1Cc1ccccc1</smiles><smiles>O=C1OCC(C2CCCCC2)=CCC1Cc1ccccc1</smiles><smiles>CC1CC=C(c2ccco2)COC1=O</smiles>

$52 \%$ yield, $>99 \%$ ee<smiles>O=C1OCC(c2ccsc2)=CCC1Cc1ccccc1</smiles><smiles>O=C1OCC(c2ccc3c(c2)OCO3)=CCC1Cc1ccccc1</smiles><smiles>O=C1OCC(/C=C/c2ccccc2)=CCC1Cc1ccccc1</smiles>

$67 \%$ yield, $99 \%$ ee<smiles>O=C(c1ccccc1)c1ccc2ccccc2c1-c1c(P)ccc2ccccc12</smiles>

L: $\mathrm{Ar}=4-\mathrm{MeC}_{6} \mathrm{H}_{4}$<smiles>Br[Mg]Br</smiles>

precat. 1: $\mathrm{Ar}=2,6-\mathrm{Et}_{2} \mathrm{C}_{6} \mathrm{H}_{3}$

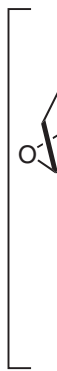

Key words

organocatalysis

$\mathrm{N}$-heterocyclic carbenes

palladium catalysis

enantioselectivity

[5+2] annulation

lactones

\section{Significance: Synthetic chemists have long} sought to combine $\mathrm{N}$-heterocyclic carbene $(\mathrm{NHC})$ organocatalysis and metal catalysis. However, because NHCs are excellent ligands for late-transition metals, the design of cooperative catalytic systems remains a formidable challenge (M. H. Wang, K. A. Scheidt Angew. Chem. Int. Ed. 2016, $55,14912)$. Reported is the first highly enantioselective [5+2] annulation between an $\mathrm{NHC}$ enolate and a $\pi$-allyl palladium intermediate through a dual catalytic process.

SYNFACTS Contributors: Victor Snieckus, Yuan Zhang (Pfizer) Synfacts 2018, 14(06), 0571 Published online: 17.05.2018 Dol: 10.1055/s-0037-1610019; Reg-No.: V05318SF
Comment: The authors expand their previous studies on NHC/Pd-based catalytic systems (J. Am. Chem. Soc. 2016, 138, 7840; ibid. 2017, $139,4443)$ by using a bidentate phosphine ligand to prevent $\mathrm{NHC}$ coordination to the active palladium catalyst. A matched combination of a chiral $\mathrm{NHC}$ and a chiral phosphine ligand promotes high levels of enantioselectivity, which has previously been a challenge for seven-membered rings due to both unfavorable entropy effects and transannular interactions (see, for example: Y.-N. Wang et al. Angew. Chem. Int. Ed. 2018, 57, 1596). 